\title{
DISCRETE FEEDBACK STABILIZATION OF SEMILINEAR CONTROL SYSTEMS
}

\author{
LARS GRÜNE
}

\begin{abstract}
For continuous time semilinear control systems with constrained control values stabilizing discrete feedback controls are discussed. It is shown that under an accessibility condition exponential discrete feedback stabilizability is equivalent to open loop exponential asymptotic null controllability. A numerical algorithm for the computation of discrete feedback controls is presented and a numerical example is discussed.
\end{abstract}

Keywords: Discrete feedback control, stabilization, semilinear control systems, Lyapunov exponents, discounted optimal control problems.

\section{INTRODUCTION}

In this paper we consider semilinear control systems of the form

$$
\left\{\begin{array}{l}
\dot{x}(t)=A(u(t)) x(t), \quad t \in \mathbb{R}, \quad x(0)=x_{0} \in \mathbb{R}^{d} \backslash\{0\} \\
u(\cdot) \in \mathcal{U}:=\{u: \mathbb{R} \rightarrow U, \text { measurable }\}, U \subset \mathbb{R}^{m} \text { compact }
\end{array}\right.
$$

where $A: \mathbb{R}^{m} \rightarrow \mathbb{R}^{d \times d}$ is Lipschitz in some open set containing $U$.

Control systems of this kind arise e.g. by linearization of nonlinear control system at a common fixed point for all control functions. In the context of this paper stabilization always means exponential asymptotic stability of the origin.

The problem of stabilization of nonlinear control systems has been considered for a long time by various authors (see e.g. Bacciotti [1] for an overview). During the last years also the more specific problem of stabilization of semilinear systems - and especially of bilinear systems which are covered by (1.1) - has attracted a great deal of attention.

Chabour et al. [5] present a Lyapunov function approach for this problem. Coller et al. [7] solve a minimum time problem in order to stabilize the system (which is possible due to the special structure of their system). In [14] stabilization is also reduced to an optimal control problem, here a discounted optimal control problem is solved in order to approximate minimal Lyapunov exponents. On the theoretical side e.g. Colonius and Kliemann [10] describe the domain of exponential asymptotical null controllability of bilinear systems, i.e. the set of points in $\mathbb{R}^{d}$ that can be asymptotically controlled to the origin exponentially fast. Although all these papers present results on the stabilization of bilinear systems general feedback stabilization

\footnotetext{
Institut für Mathematik, Universität Augsburg, Universitätsstr. 8, 86135 Augsburg, Germany. E-mail: Lars.Gruene@Math.Uni-Augsburg.de.

Received by the journal December 6, 1995. Accepted for publication June 18, 1996.

This work has been supported by DFG-Grant Co 124/12-1.

(C) Société de Mathématiques Appliquées et Industrielles.
} 
techniques seem to be missing up to now. The results cited above make either use of special structures of the systems considered or are focused on open loop asymptotic null controllability rather than on feedbacks.

In this paper we will restrict ourselves to the case, where the system is exponentially asymptotically null controllable by an open loop control for each initial value $x_{0} \in \mathbb{R}^{d}$. The question that arises then is whether under this condition there exists a feedback control such that the corresponding closed loop system is exponentially stable. In general this is not possible by using a continuous feedback law, cp. [2]. Hence we will use a more general feedback concept which we will call discrete feedback controls.

In mathematical control theory discrete feedback controls have been investigated by various authors: Hermes [15], [16] makes use of this construction under the name of modified feedback control applying Lie algebraic methods to the stabilization problem. Sontag applies the same idea using the terminology sampled feedback or sample-and-hold control in connection with nonlinear regulation [20] and also with stabilization using neural networks [21] (the term "sampling" has its origin in engineering). A similar concept can be found in the context of dynamic game theory (see e.g. Krasovski1 and Subbotin [18]); there the value of a game is defined via discrete control functions (called step-by-step control) where the size of the discretization step tends to 0 . Inspired by this technique Clarke, Ledyaev, Sontag and Subbotin show in a recent work [6] using Lyapunov functions that asymptotic controllability implies stabilizability of nonlinear control systems by sampled feedbacks when the discretization step (or sampling rate) tends to 0 .

The construction made in this paper is based on another concept introduced by Lyapunov, namely the Lyapunov exponents. It has its origin in the numerical considerations discussed in [14] which deal with the numerical calculation of open loop control functions that control (1.1) asymptotically to the origin. Like in many numerical algorithms a discretization of (1.1) is needed in order to apply the algorithm from [14]. Hence it seems natural to consider the discrete time system obtained from (1.1) by discretization in time. The discrete feedback discussed here can then be interpreted as a feedback for this discrete time system applied to the continuous time system. In contrast to the result by Clarke et al. here we obtain stabilizability using discrete feedback controls with fixed discretization step size. Moreover we will give a numerical algorithm to calculate the stabilizing discrete feedback control.

The organization of this paper is as follows. In the second section we will cite previous results on asymptotic null controllability of semilinear control systems, define Lyapunov exponents and the discounted optimal control problem and show the relation between these two concepts. The only assumption that will be made on the system is an accessibility condition as described in [17].

In the third section discrete feedback controls will be defined and it will be shown that they are optimal control strategies for the discounted optimal control problem and discrete time control systems.

Section 4 then applies this result to the stabilization problem. We will show that exponential open loop asymptotic null controllability for all $x_{0} \in$ 
$\mathbb{R}^{d}$ is equivalent to the existence of an exponentially stabilizing discrete feedback.

In Section 5 a numerical algorithm for the computation of these stabilizing discrete feedback controls is developed and finally in Section 6 a numerical example is presented.

\section{Problem Setup and PREliminary Results}

We consider semilinear control systems of the form

$$
\left\{\begin{array}{l}
\dot{x}(t)=A(u(t)) x(t), \quad t \in \mathbb{R}, \quad x(0)=x_{0} \in \mathbb{R}^{d} \backslash\{0\} \\
u(\cdot) \in \mathcal{U}:=\{u: \mathbb{R} \rightarrow U, \text { measurable }\}, U \subset \mathbb{R}^{m} \text { compact }
\end{array}\right.
$$

where $A: \mathbb{R}^{m} \rightarrow \mathbb{R}^{d \times d}$ is Lipschitz in some open set containing $U$. Let $x\left(t, x_{0}, u(\cdot)\right)$ denote the solution of $(2.1)$ for an initial value $x_{0}$ and a control function $u(\cdot) \in \mathcal{U}$.

In order to characterize the (open-loop) exponential behaviour of (2.1) we define the Lyapunov exponent $\lambda\left(x_{0}, u(\cdot)\right)$ by

$$
\lambda\left(x_{0}, u(\cdot)\right):=\limsup _{t \rightarrow \infty} \frac{1}{t} \ln \left\|x\left(t, x_{0}, u(\cdot)\right)\right\|,
$$

and the infimal Lyapunov exponent with respect to the control by

$$
\lambda^{*}\left(x_{0}\right):=\inf _{u(\cdot) \in \mathcal{U}} \lambda\left(x_{0}, u(\cdot)\right) .
$$

The following assertion is an easy consequence from the definition of the Lyapunov exponent:

For all $x_{0} \in \mathbb{R}^{d}, x_{0} \neq 0$ there exists a control function $u_{x_{0}}(\cdot) \in \mathcal{U}$ such that $x\left(t, x_{0}, u_{x_{0}}(\cdot)\right)$ converges to the origin exponentially fast if and only if $\lambda^{*}\left(x_{0}\right)<0$ for all $x_{0} \in \mathbb{R}^{d} \backslash\{0\}$.

In the following we will restrict ourselves to the case $\lambda^{*}\left(x_{0}\right)<0$ for all $x_{0} \in \mathbb{R}^{d} \backslash\{0\}$ and turn to the question if this is also a (necessary and/or sufficient) condition for the existence of a feedback controller that stabilizes (2.1). Instead of using "classical" feedbacks we will define discrete feedback controls and show that - under an accessibility condition - the existence of an exponentially stabilizing discrete feedback control is equivalent to this condition. Furthermore we will present a numerical algorithm to compute discrete feedback controls.

In the rest of this section we will collect the facts known about semilinear control systems.

As a consequence of the semilinear structure of (2.1) it follows that $\lambda(x, u(\cdot))=\lambda\left( \pm \frac{x}{\|x\|}, u(\cdot)\right)$. Hence for the analysis of the Lyapunov exponents it is sufficient to consider (2.1) projected to the real projective space $\mathbb{P}^{d-1}$. We will represent $\mathbb{P}^{d-1}$ by the sphere $\mathbb{S}^{d-1}$, where opposite points $-s$ and $s$ are identified. Using the chain rule it is easy to verify that the projected system $s(t):=\frac{x(t)}{\|x(t)\|}$ can be written as

$$
\begin{aligned}
& \dot{s}(t)=h(s(t), u(t)), \\
& \text { where } h(s, u)=\left[A(u)-s^{t} A(u) s \quad \mathrm{Id}\right] s
\end{aligned}
$$

Let $\varphi\left(t, s_{0}, u(\cdot)\right)$ denote the solution of $(2.4)$ with initial value $s_{0} \in \mathbb{S}^{d-1}$. The Lyapunov exponent for any initial value $x_{0} \in \mathbb{R}^{d}$ with $s_{0}=\frac{x_{0}}{\left\|x_{0}\right\|}$ can 
then be expressed as (see also [9, Section 2])

$$
\begin{aligned}
& \lambda\left(x_{0}, u(\cdot)\right)=\limsup \sup _{t \rightarrow \infty} \frac{1}{t} \int_{0}^{t} g\left(\varphi\left(t, s_{0}, u(\cdot)\right), u(t)\right) d t, \\
& \text { where } g(s, u)=s^{t} A(u) s .
\end{aligned}
$$

The following assumption assures local accessibility of (2.4), i.e. that the reachable set for any point up to any time $t>0$ has nonvoid interior (cp. $[17]$ ).

Let $L$ denote the Lie-algebra generated by the vector fields $h(\cdot, u), u \in U$. Let $\Delta_{L}$ denote the distribution generated by $L$ in $T \mathbb{P}^{d-1}$, the tangent bundle of $\mathbb{P}^{d-1}$. Assume that

$$
\operatorname{dim} \Delta_{L}(p)=\operatorname{dim} \mathbb{P}^{d-1}=d-1 \text { for all } p \in \mathbb{P}^{d-1} .
$$

Under this condition there exists a unique invariant control set $C$ with nonvoid interior of the projected system, i.e. a region of complete controllability which no trajectory can leave. Furthermore for any two points $p_{1} \in \mathbb{P}^{d-1}, p_{2} \in \operatorname{int} C$ there exists a control function $u_{p_{1}, p_{2}}(\cdot) \in \mathcal{U}$ and a time $t$ such that $\varphi\left(t, p_{1}, u_{p_{1}, p_{2}}(\cdot)\right)=p_{2}$. (For a comprehensive analysis of the controllability structure of projected semilinear systems see [8].) Using this control $u_{p_{1}, p_{2}}(\cdot)$ for any $\varepsilon>0$ we can define a control function that steers $p_{1}$ to $p_{2}$ and then realizes the minimal Lyapunov exponent for $p_{2}$ up to $\varepsilon$. This yields (cp. also [14, Proposition 2.6])

$$
\lambda^{*}\left(p_{1}\right) \leq \lambda^{*}\left(p_{2}\right) \text {. }
$$

Furthermore by symmetry $\lambda^{*}$ is constant on int $C$.

Putting this together yields that

$$
\tilde{\kappa}:=\max _{x_{0} \in \mathbb{R}^{d} \backslash\{0\}} \lambda^{*}\left(x_{0}\right)
$$

exists, $\tilde{\kappa}$ is attained on $\operatorname{int} C$ and it holds that

$$
\begin{aligned}
& \lambda^{*}\left(x_{0}\right)<0 \text { for all } x_{0} \in \mathbb{R}^{d} \backslash\{0\} \\
& \text { if and only if } \tilde{\kappa}<0 .
\end{aligned}
$$

In order to construct a discrete feedback law we approximate (2.5) by a discounted optimal value function $v_{\delta}$ with discount rate $\delta$ defined by

$$
\begin{aligned}
& v_{\delta}\left(s_{0}\right):=\inf _{u(\cdot) \in \mathcal{U}} J_{\delta}\left(s_{0}, u(\cdot)\right), \\
& \text { where } J_{\delta}\left(s_{0}, u(\cdot)\right):=\int_{0}^{\infty} e^{-\delta t} g\left(\varphi\left(t, s_{0}, u(\cdot)\right), u(t)\right) d t .
\end{aligned}
$$

The following results from [14, Theorem 2.1 and Theorem 2.11] are crucial for this approximation.

Theorem 2.1. Consider a semilinear system (2.1) and its projection (2.4) satisfying $(H)$. Then

1. $\delta v_{\delta} \rightarrow \lambda^{*}$ uniformly on compact subsets of intC as $\delta \rightarrow 0$.

2. $\sup \delta v_{\delta}(s) \rightarrow \tilde{\kappa}$ as $\delta \rightarrow 0$. $s \in \mathbb{P}^{d-1}$

3. Let $u(\cdot) \in \mathcal{U}$ and $\alpha \in \mathbb{R}$ such that the following shift condition is satisfied:

$$
\delta J_{\delta}(\varphi(t, s, u(\cdot)), u(t+\cdot)) \leq \alpha \text { for all } t \geq 0 .
$$


Then $\lambda(s, u(\cdot)) \leq \alpha$.

The proof of this theorem is essentially based on two arguments: In the first step the relation between averaged functionals (which appear in the definition of the Lyapunov exponent) and discounted functionals has to be investigated. In the second step a controllability property similar to the one cited above but in uniform time is used to obtain the assertions.

Theorem 2.1 states that the maximum (over all initial values) of the infimal (over all control functions) Lyapunov exponents of (2.1) can be approximated by a discounted optimal control problem on the projective space. Furthermore any control function satisfying the shift condition as defined in (iii) will yield a Lyapunov exponent smaller than or equal to $\alpha$ when applied to (2.1). Hence the next section is devoted to the construction of a discrete feedback law that yields approximately optimal solutions of (2.4) with respect to $(2.7)$.

\section{Discrete Feedback Control}

We will now give the definition of discrete feedback control.

Definition 3.1. (Discrete feedback control) A discrete feedback control for the system $(2.1)$ is a function $F: \mathbb{R}^{d} \rightarrow U$ in connection with a time step $h>0$ that is applied to $(2.1)$ via

$$
\dot{x}(t)=A\left(F\left(x\left(\left[\frac{t}{h}\right] h\right)\right)\right) x(t)
$$

where $[r]$ denotes the largest integer less or equal $r \in \mathbb{R}$.

REMARK 3.2. The following interpretation gives the motivation for the name "discrete feedback". For a given time step $h>0$ and constant control values $u \in U$ denote by $G: \mathbb{R}^{d} \times U \rightarrow \mathbb{R}^{d}$ the solution of (2.1) at the time $h$, i.e. $G\left(x_{0}, u\right):=x\left(h, x_{0}, u\right)$. This defines a discrete time control system via

$$
x_{i+1}:=G\left(x_{i}, u_{i}\right), \quad\left(u_{i}\right)_{i \in \mathbb{N}} \in U^{\mathbb{N}} .
$$

The discrete feedback as defined in Definition 3.1 can now be interpreted as a feedback for the discrete time system (3.1).

In particular this interpretation shows that for any discrete feedback $F$ and any initial value $x_{0} \in \mathbb{R}^{d}$ there exists a unique solution which will be denoted by $x_{F}\left(t, x_{0}\right)$. Note that no regularity conditions on $F-$ not even continuity - are necessary in order to obtain existence and uniqueness of the corresponding solution. We will also consider discrete feedbacks $F_{\mathbb{P}}$ for the projected system; the corresponding solutions are again unique and will be denoted by $\varphi_{F_{\mathrm{p}}}\left(t, s_{0}\right)$.

System (3.1) corresponds to (2.1) where only control functions $u_{h}$ constant on intervals with uniform length $h>0$ are admissible. This system can also be projected to $\mathbb{P}^{d-1}$. We will now investigate how the value of $v_{\delta}$ (corresponding to the projected system) changes if we restrict ourselves to this class of control functions. Define

$$
\begin{aligned}
& \mathcal{U}_{h}:=\left\{u_{h}: \mathbb{R} \rightarrow U|u|_{[i h,(i+1) h)} \equiv \text { const }\right\} \\
& \text { ESA IM: Cocv, September 1996, Vol. 1, PP. 207-224 }
\end{aligned}
$$


and

$$
v_{\delta}^{h}(s):=\inf _{u(\cdot) \in \mathcal{U}_{h}} J_{\delta}(s, u(\cdot)) .
$$

A basic property of the optimal value functions of discounted optimal control problems is Bellman's optimality principle (see e.g. [19, Theorem 1.2]): For all $\tau>0$ it holds that

$$
v_{\delta}(s)=\inf _{u(\cdot) \in \mathcal{U}} \int_{0}^{\tau} e^{-\delta t} g(\varphi(t, s, u(\cdot)), u(t)) d t+e^{-\delta \tau} v_{\delta}(\varphi(\tau, s, u(\cdot)) .
$$

The value function $v_{\delta}^{h}$ satisfies a similar equation which is proved similar to [3, Proposition 1.1]. It holds that

$$
v_{\delta}^{h}(s)=\inf _{u \in U} \int_{0}^{h} e^{-\delta t} g(\varphi(t, s, u), u) d t+e^{-\delta h} v_{\delta}^{h}(\varphi(\tau, s, u)) .
$$

The main difference between these two equalities lies in the fact that in the second one control values instead of control functions are considered. The following theorem shows the convergence for $h \rightarrow 0$.

Theorem 3.3. Consider the control system (2.4) and the optimal value functions $v_{\delta}$ and $v_{\delta}^{h}$. Then

$$
\left\|v_{\delta}-v_{\delta}^{h}\right\|_{\infty}<C h^{\frac{\gamma}{2}}
$$

where $C>0$ is some constant, $\gamma=\frac{L_{h}}{\delta}$ for $\delta<L_{h}$ and $L_{h}$ is the Lipschitz constant of h from (2.4) with respect to $s$. Furthermore $v_{\delta}$ and $v_{\delta}^{h}$ are Hoelder continuous with exponent $\gamma$.

Proof: Similar to [4, Theorem 4.1], where we use the metric on $\mathbb{S}^{d-1}$ induced by the norm on $\mathbb{R}^{d}$.

REMARK 3.4. Under stronger assumptions on the system (i.e. control affine systems, convex control range $U$ ) it is possible to obtain the same estimate with $\gamma$ instead of $\frac{\gamma}{2}$, see $[12$, Section 5$]$.

The discrete feedback can now be constructed as follows:

Definition 3.5. Fix $h>0$ and define $F_{\mathbb{P}}: \mathbb{P}^{d-1} \rightarrow U$ by: For every $s \in$ $\mathbb{P}^{d-1}$ choose a value $u \in U$ such that

$$
\int_{0}^{h} e^{-\delta t} g(\varphi(t, s, u), u) d t+e^{-\delta h} v_{\delta}^{h}(\varphi(h, s, u))
$$

becomes minimal and let $F_{\mathbb{P}}(s):=u$.

The function $F_{\mathbb{P}}$ from the definition above may not be unique, however the existence of a control value $F_{\mathbb{P}}(s)$ with the desired property is always guaranteed by the continuity of $g, v_{\delta}^{h}$ and $u \mapsto \varphi(h, s, u)$ and the compactness of $U$.

A discrete feed back for the non projected system (2.1) can easily be derived from $F_{\mathbb{P}}$ by defining $F(x):=F_{\mathbb{P}}(x /\|x\|)$.

The following theorem shows that this discrete feedback is indeed an optimal control strategy for $v_{\delta}^{h}$. 
Theorem 3.6. Consider the system (2.4) and the optimal value function $v_{\delta}^{h}$. Let $F_{\mathbb{P}}$ be the discrete feedback from Definition 3.5. Then for any initial value $s_{0} \in \mathbb{P}^{d-1}$ it holds that

$$
\int_{0}^{\infty} e^{-\delta t} g\left(\varphi_{F_{\mathbb{P}}}\left(t, s_{0}\right), F_{\mathbb{P}}\left(\varphi_{F_{\mathbb{P}}}\left(\left[\frac{t}{h}\right] h, s_{0}\right)\right)\right) d t=v_{\delta}^{h}\left(s_{0}\right) .
$$

Proof: Abbreviate

$$
K\left(s_{0}\right):=\int_{0}^{\infty} e^{-\delta t} g\left(\varphi_{F_{\mathbb{D}}}\left(t, s_{0}\right), F_{\mathbb{P}}\left(\varphi_{F_{\mathbb{P}}}\left(\left[\frac{t}{h}\right] h, s_{0}\right)\right)\right) d t .
$$

Then the identity

$$
K\left(s_{0}\right)=\int_{0}^{h} e^{-\delta t} g\left(\varphi_{F_{\mathbb{P}}}\left(t, s_{0}\right), F_{\mathbb{P}}\left(s_{0}\right)\right) d t+e^{-\delta h} K\left(\varphi_{F_{\mathbb{P}}}\left(h, s_{0}\right)\right)
$$

is obvious. On the other hand by the definition of $F_{\mathbb{P}}$ it holds that

$$
\begin{gathered}
v_{\delta}^{h}\left(s_{0}\right)=\inf _{u \in U}\left\{\int_{0}^{h} e^{-\delta t} g\left(\varphi\left(t, s_{0}, u\right), u\right) d t+e^{-\delta h} v_{\delta}^{h}\left(\varphi\left(\tau, s_{0}, u\right)\right)\right\} \\
=\int_{0}^{h} e^{-\delta t} g\left(\varphi\left(t, s_{0}, F_{\mathbb{P}}\left(s_{0}\right)\right), F_{\mathbb{P}}\left(s_{0}\right)\right) d t \\
+e^{-\delta h} v_{\delta}^{h}\left(\varphi\left(\tau, s, F_{\mathbb{P}}\left(s_{0}\right)\right)\right) .
\end{gathered}
$$

Subtracting (3.4) from (3.3) yields

$$
\left|K\left(s_{0}\right)-v_{\delta}^{h}\left(s_{0}\right)\right|=e^{-\delta h}\left|K\left(\varphi_{F_{\mathbb{P D}}}\left(h, s_{0}\right)\right)-v_{\delta}^{h}\left(\varphi_{F_{\mathrm{TP}}}\left(h, s_{0}\right)\right)\right|
$$

which implies

$$
\sup _{s \in \mathbb{P}}\left|K(s)-v_{\delta}^{h}(s)\right| \leq e^{-\delta h} \sup _{s \in \mathbb{P}}\left|K(s)-v_{\delta}^{h}(s)\right| .
$$

This implies the assertion since $e^{-\delta h}<1$.

Corollary 3.7. For any $\varepsilon>0$ there exists $h>0$ such that the solution $\varphi_{F_{\mathbb{R}}}\left(t, s_{0}\right)$ corresponding to the discrete feedback from Definition 3.5 satisfies

$$
\left|\int_{0}^{\infty} e^{-\delta t} g\left(\varphi_{F_{\mathbb{P}}}\left(t, s_{0}\right), F_{\mathbb{P}}\left(\varphi_{F_{\mathbb{P}}}\left(\left[\frac{t}{h}\right] h, s_{0}\right)\right)\right) d t-v_{\delta}\left(s_{0}\right)\right|<\varepsilon
$$

for all $s_{0} \in \mathbb{P}^{d-1}$.

Proof: Follows immediately from Theorem 3.3 and Theorem 3.6.

This corollary states that a discrete feedback can be used to obtain approximately optimal solutions for the discounted optimal control problem.

REMARK 3.8. Although for application and numerical analysis the discrete feedback control has very nice features (as shown in the next sections), from the mathematical point of view it would nevertheless be interesting to know the properties of the closed loop system

$$
\dot{x}=A(F(x)) x \text {. }
$$


Since $F$ is typically discontinuous the existence and uniqueness of trajectories is not clear. It is also not known whether this system preserves the optimality properties of the discrete feedback system.

Clearly this question leads to the problem of the existence of optimal feedback controls for discounted optimal control problems. If one uses dynamic programming in order to obtain optimal open loop control functions $u_{h}(\cdot)$ it has been shown that for $h \rightarrow 0$ there exists at least a weakly convergent subsequence $u_{h_{n}}(\cdot)$ for $h_{n} \rightarrow 0$, see [4, Theorem 5.1]. It is still an open question whether a similar construction can be obtained for the optimal feedback control.

\section{Stabilization using discrete FeEdBack Control}

In this section we will apply the discrete feedback as defined in the previous section to the stabilization problem. Theorem 2.1 (ii) and Corollary $3.7 \mathrm{imply}$ that for any $\varepsilon>0$ there exists $h>0$ and a discrete feedback such that

$$
\delta \int_{0}^{\infty} e^{-\delta t} g\left(\varphi_{F_{\mathbb{p}}}\left(t, s_{0}\right), F_{\mathbb{P}}\left(\varphi_{F_{\mathbb{D}}}\left(\left[\frac{t}{h}\right] h, s_{0}\right)\right)\right) d t<\tilde{\kappa}+\varepsilon .
$$

Hence it remains to show that the corresponding trajectories also satisfy the shift property to meet the assumption of Theorem 2.1 (iii).

LemMa 4.1. Assume there exists $h>0$, a discrete feedback $F_{\mathbb{P}}$ and a constant $\alpha \in \mathbb{R}$ such that

$$
\int_{0}^{\infty} e^{-\delta t} g\left(\varphi_{F_{\mathbb{P}}}\left(t, s_{0}\right), F_{\mathbb{P}}\left(\varphi_{F_{\mathbb{P}}}\left(\left[\frac{t}{h}\right] h, s_{0}\right)\right)\right) d t \leq \alpha
$$

for all $s_{0} \in \mathbb{P}^{d-1}$.

Then there exists a constant $B>0$ independent of $h$ such that for all $T \geq 0$ it holds that

$$
\int_{T}^{\infty} e^{-\delta(t-T)} g\left(\varphi_{F_{\mathbb{P}}}\left(t, s_{0}\right), F_{\mathbb{P}}\left(\varphi_{F_{\mathbb{D}}}\left(\left[\frac{t}{h}\right] h, s_{0}\right)\right)\right) d t \leq \alpha+B h .
$$

Proof: For $T=i h, i \in \mathbb{N}$ the assertion follows directly from the assumption. For arbitrary $T>0$ observe that $g(s, u)$ is bounded by a constant $M_{g}$. Choosing $0<\tau<h$ such that $T+\tau=i h$ for some $i \in \mathbb{N}$ it follows that

$$
\begin{aligned}
& \int_{T}^{\infty} e^{-\delta(t-T)} g\left(\varphi_{F_{\mathbb{D}}}\left(t, s_{0}\right), F_{\mathbb{P}}\left(\varphi_{F_{\mathbb{D}}}\left(\left[\frac{t}{h}\right] h, s_{0}\right)\right)\right) d t \\
= & \int_{T}^{T+\tau} e^{-\delta(t-T)} g\left(\varphi_{F_{\mathbb{D}}}\left(t, s_{0}\right), F_{\mathbb{P}}\left(\varphi_{F_{\mathbb{P}}}\left(\left[\frac{t}{h}\right] h, s_{0}\right)\right)\right) d t \\
& +\int_{T+\tau}^{\infty} e^{-\delta(t-T)} g\left(\varphi_{F_{\mathbb{D}}}\left(t, s_{0}\right), F_{\mathbb{P}}\left(\varphi_{F_{\mathbb{P}}}\left(\left[\frac{t}{h}\right] h, s_{0}\right)\right)\right) d t \\
\leq & \tau M_{g}+e^{-\delta \tau} \alpha .
\end{aligned}
$$


Now the assertion follows by Taylor expansion of $e^{-\delta \tau}$ since $\tau<h$ (note that $\alpha$ may be negative).

Now we have collected all the facts to prove the following theorem about the existence of stabilizing discrete feedbacks.

Theorem 4.2. Consider a semilinear control system (2.1) satisfying $(H)$. Then for any $\varepsilon>0$ there exists a $h^{\prime}>0$ such that for any $h \leq h^{\prime}$ there exists a discrete feedback $F: \mathbb{R}^{d} \rightarrow U$ such that

$$
\limsup _{t \rightarrow \infty} \frac{1}{t} \ln \left\|x_{F}\left(t, x_{0}\right)\right\| \leq \tilde{\kappa}+\varepsilon .
$$

Proof: Using Theorem 2.1(ii) and Corollary 3.7 it follows that there exists $h^{\prime \prime}>0$ such that for any $h \leq h^{\prime \prime}$ there exists a discrete feedback $F_{\mathbb{P}}$ that satisfies the assumptions of Lemma 4.1 with $\alpha=\tilde{\kappa}+\varepsilon / 2$. Choosing $h^{\prime} \leq h^{\prime \prime}$ sufficiently small Lemma 4.1 with $h \leq h^{\prime}$ yields the assumption of Theorem 2.1 (iii) with $\alpha=\tilde{\kappa}+\varepsilon$. Now the assertion follows by defining $F(x):=$ $F_{\mathbb{P}}(x /\|x\|)$.

In particular we obtain a result about exponential stabilization of semilinear control systems with discrete feedback:

Theorem 4.3. Consider a semilinear control system (2.1) satisfying $(H)$. Then there exists $a h>0$ and a discrete feedback that steers (2.1) to the origin exponentially fast for all initial values $x_{0} \in \mathbb{R}^{d} \backslash\{0\}$ if and only if $\lambda^{*}\left(x_{0}\right)<0$ for all $x_{0} \in \mathbb{R}^{d} \backslash\{0\}$.

PRoOF: " $\Rightarrow$ ": The existence of a discrete feedback immediately implies the existence of a measurable open loop control for any initial value $x_{0} \in \mathbb{R}^{d}$ steering (2.1) to the origin exponentially fast. Hence $\lambda^{*}\left(x_{0}\right)<0$ is implied.

" $\Leftarrow$ ": Assume $\lambda^{*}\left(x_{0}\right)<0$ for all $x_{0} \in \mathbb{R}^{d} \backslash\{0\}$. Then (2.6) implies $\tilde{\kappa}<0$ and hence there exists $\varepsilon>0$ such that $\tilde{\kappa}+\varepsilon<0$. Now by Theorem 4.2 there exists a discrete feedback with the desired properties.

REMARK 4.4. Note that the equivalence (2.6) plays an important role in the proof of this theorem: It prevents the existence of a sequence $\left(x_{i}\right)_{i \in \mathbb{N}}$ such that $\lambda^{*}\left(x_{i}\right) \nearrow 0$. In this case the conclusion used in the second part of the proof would not be possible.

\section{Numerical CAlCulation of THE Discrete FEEDBACK}

The numerical method proposed follows the ideas described in [14] which are based on a discretization scheme by I. Capuzzo Dolcetta, H. Ishii and M. Falcone [4], [11] and [3]. However, in order to construct the discrete feedback control and ensure convergence we have to modify this algorithm. Some of the ideas presented here have also been investigated by M. Falcone and R. Ferretti [12] in the case of large discount rates.

In fact three steps of discretization apply here:

(i) The measurable control functions in $\mathcal{U}$ are replaced by the piecewise constant control functions in $\mathcal{U}_{h}$ with uniform step size $h$.

(ii) The integral

$$
\int_{0}^{\infty} e^{-\delta \tau} g\left(\varphi\left(\tau, s, u_{h}(\cdot)\right), u_{h}(\tau)\right) d \tau
$$


is replaced by

$$
h \sum_{i=0}^{\infty} \beta^{i} g\left(\varphi\left(i h, s, u_{h}(\cdot)\right), u_{h}(i h)\right)
$$

with $\beta=1-\delta h$.

(iii) The trajectory $\varphi\left(t, s, u_{h}(\cdot)\right)$ of the system is replaced by a numerical approximation $\tilde{\varphi}_{i}\left(s, u_{h}(\cdot)\right)$.

The discretization error of the first step has already been stated in Theorem 3.3. In the next two lemmas we will investigate the rates of convergence for the discretization of the integration.

LEMMA 5.1. Let $\delta>0$ and $h>0$. Let $g: \mathbb{R} \rightarrow \mathbb{R}$ be a real valued function bounded by $M_{g}$ and Lipschitz on each interval $[i h,(i+1) h), i \in \mathbb{N}_{0}$ with Lipschitz constant $L_{g}$. Then

$$
\left|\int_{0}^{\infty} e^{-\delta t} g(t) d t-h \sum_{i=0}^{\infty} e^{-\delta h i} g(h i)\right| \leq \frac{e^{\delta h}\left(L_{g}+\delta M_{g}\right)}{\delta} h
$$

Proof: For $t_{1}, t_{2} \in[i h,(i+1) h), i \in \mathbb{N}_{0}$ it holds that

$$
\begin{aligned}
& \left|e^{-\delta t_{1}} g\left(t_{1}\right)-e^{-\delta t_{2}} g\left(t_{2}\right)\right| \\
\leq & \left|e^{-\delta t_{1}} g\left(t_{1}\right)-e^{-\delta t_{2}} g\left(t_{1}\right)\right|+\left|e^{-\delta t_{2}} g\left(t_{1}\right)-e^{-\delta t_{2}} g\left(t_{2}\right)\right| \\
\leq & \left(\delta M_{g}+L_{g}\right)\left|t_{1}-t_{2}\right| .
\end{aligned}
$$

This implies for all $\tau \in[i h,(i+1) h), i \in \mathbb{N}_{0}$

$$
\left|\int_{i h}^{(i+1) h} e^{-\delta t} g(t) d t-h e^{-\delta \tau} g(\tau)\right| \leq e^{-\delta i h}\left(L_{g}+\delta M_{g}\right) h^{2}
$$

and hence

$$
\begin{aligned}
& \left|\int_{0}^{\infty} e^{-\delta t} g(t) d t-h \sum_{i=0}^{\infty} e^{-\delta h i} g(h i)\right| \\
\leq & \sum_{i=0}^{\infty} e^{-\delta h i}\left(L_{g}+\delta M_{g}\right) h^{2} \\
\leq & e^{\delta h} \int_{0}^{\infty} e^{-\delta t}\left(L_{g}+\delta M_{g}\right) h d t=\frac{e^{\delta h}\left(L_{g}+\delta M_{g}\right)}{\delta} h .
\end{aligned}
$$

LeMMA 5.2. Let $\beta:=1-\delta h$. Under the assumptions of Lemma 5.1 it holds that

$$
\left|h \sum_{i=0}^{\infty} e^{-\delta i h} g(i h)-h \sum_{i=0}^{\infty} \beta^{i} g(i h)\right| \leq C M_{g} h
$$

for a constant $C>0$. 
Proof: It holds that

$$
\begin{aligned}
\left|h \sum_{i=0}^{\infty} e^{-\delta i h} g(i h)-h \sum_{i=0}^{\infty} \beta^{i} g(i h)\right| & \leq\left|h M_{g}\left(\frac{1}{1-e^{-\delta h}}-\frac{1}{\delta h}\right)\right| \\
& =\left|h M_{g} \frac{e^{-\delta h}-(1-\delta h)}{\delta h\left(1-e^{-\delta h}\right)}\right| \\
& \leq\left|h M_{g} C\right|
\end{aligned}
$$

where the last inequality is seen by Taylor expansion of $e^{-\delta h}$.

These two lemmas show that the discretization error for the integration is of linear order in $h$.

We will now turn to the approximation of the trajectories by a numerical algorithm. For this purpose we assume that for any control function $u_{h}(\cdot) \in$ $\mathcal{U}_{h}$ the function $\tilde{\varphi}_{i}(s, u)$ is a numerical approximation of the continuous time trajectory satisfying

$$
\left\|\tilde{\varphi}_{1}\left(s, u_{h}(\cdot)\right)-\varphi\left(h, s, u_{h}(\cdot)\right)\right\| \leq \varepsilon(h) h
$$

with $\varepsilon(h) \searrow 0$ as $h \rightarrow 0$ and

$$
\left\|\tilde{\varphi}_{i}\left(s_{1}, u_{h}(\cdot)\right)-\tilde{\varphi}_{i}\left(s_{2}, u_{h}(\cdot)\right)\right\| \leq e^{L i h}\left\|s_{1}-s_{2}\right\|
$$

for all $i \in \mathbb{N}, s, s_{1}, s_{2} \in \mathbb{P}^{d-1}$, all $u_{h}(\cdot) \in \mathcal{U}_{h}$ and a constant $L>0$. In the numerical analysis of ordinary differential equations these are the two standard conditions consistency and Lipschitz continuity. If $\varepsilon(h)=O\left(h^{p}\right)$ for some $p \in \mathbb{N}$ the scheme is called consistent with order $p$. By induction one can prove that these assumptions imply

$$
\left\|\tilde{\varphi}_{i}\left(s, u_{h}(\cdot)\right)-\varphi\left(i h, s, u_{h}(\cdot)\right)\right\| \leq \frac{\varepsilon(h)}{L}\left(e^{L i h}-1\right) .
$$

The following result shows what happens in the third step when the original system with piecewise constant control functions is approximated by this numerical algorithm.

THEOREM 5.3. Let $\delta>0$ and $\tilde{\varphi}_{i}\left(s, u_{h}(\cdot)\right)$ be a numerical approximation of the continuous time system satisfying (5.1)-(5.3). Let

$$
\tilde{J}_{\delta}^{h}\left(s, u_{h}(\cdot)\right):=h \sum_{i=0}^{\infty} \beta^{i} g\left(\tilde{\varphi}_{i}\left(s, u_{h}(\cdot)\right), u_{h}(i h)\right), \tilde{v}_{\delta}^{h}(s):=\inf _{u_{h}(\cdot) \in \mathcal{U}_{h}} \tilde{J}_{\delta}^{h}\left(s, u_{h}(\cdot)\right)
$$

and let $v_{\delta}^{h}$ be the optimal value function from (3.2). Then $\tilde{v}_{\delta}^{h}$ is Hoelder continuous with exponent $\gamma$ and

$$
\left|v_{\delta}^{h}(s)-\tilde{v}_{\delta}^{h}(s)\right| \leq C\left(\varepsilon(h)^{\gamma}+h\right)
$$

for all $s \in \mathbb{P}^{d-1}$ with $\gamma=\frac{\delta}{L}$ and $C=O\left(\frac{1}{\delta}\right)$ for $\delta<L$.

Proof: Follows immediately using [4, Lemma 4.1] and the preceding lemmas.

The value function $\tilde{v}_{\delta}^{h}$ is still defined for every point $s \in \mathbb{P}^{d-1}$. For the numerical calculation we have to restrict ourselves to a finite set of points in $\mathbb{P}^{d-1}$, i.e. using a suitable parametrization of $\mathbb{P}^{d-1}$ we have to compute an approximation on a grid covering some $\Omega \subset \mathbb{R}^{d-1}$. This approximation 
can be calculated e.g. as described in [11], [14] or [13]. We will now assume that $\tilde{v}_{\delta}^{a}$ gives such an approximation for $\tilde{v}_{\delta}^{h}$ satisfying

$$
\left|\tilde{v}_{\delta}^{a}(s)-\tilde{v}_{\delta}^{h}(s)\right| \leq \eta \text { for all } s \in \mathbb{P}^{d-1}
$$

and use $\tilde{v}_{\delta}^{a}$ in order to construct an approximately optimal feedback law for the discrete time system.

Lemma 5.4. Let $\tilde{F}_{\mathbb{P}}: \mathbb{P}^{d-1} \rightarrow U$ be a discrete feedback law such that

$$
h g\left(s, \tilde{F}_{\mathbb{P}}(s)\right)+\beta \tilde{v}_{\delta}^{a}\left(\tilde{\varphi}_{i}\left(s, \tilde{F}_{\mathbb{P}}(s)\right)\right)=\min _{u \in U}\left\{h g(s, u)+\beta \tilde{v}_{\delta}^{a}\left(\tilde{\varphi}_{i}(s, u)\right)\right\}
$$

holds for every $s \in \mathbb{P}^{d-1}$. Let $\tilde{\varphi}_{\tilde{F}_{\mathrm{T}}, i}(s)$ denote the corresponding solution using this discrete feedback and the numerical approximation of the trajectories. Then

$$
\| h \sum_{i=0}^{\infty} \beta^{i} g\left(\tilde{\varphi}_{\tilde{F}_{\mathbb{P}}, i}(s), \tilde{F}_{\mathbb{P}}\left(\tilde{\varphi}_{\tilde{F}_{\mathbb{P}}, i}(s)\right)-\tilde{v}_{\delta}^{a}(x) \| \leq \frac{2 \eta}{\delta h} \text { for all } s \in \mathbb{P}^{d-1} .\right.
$$

Proof: Property (5.4) yields that

$$
\tilde{v}_{\delta}^{a}(s)=h g\left(x, \tilde{F}_{\mathbb{P}}(s)\right)+\beta \tilde{v}_{\delta}^{a}\left(\tilde{\varphi}_{\tilde{F}_{\mathbb{P}}, i}(s)\right)+2 e(s)
$$

for all $s \in \mathbb{P}^{d-1}$ where $|\eta(s)|<\eta$. This immediately implies the assertion.

The following proposition gives an estimate for the error made when the discrete feedback law $\tilde{F}_{\mathbb{P}}$ is applied to (2.4) according to Definition 3.1.

Proposition 5.5. The solutions $\varphi_{\tilde{F}_{\mathrm{p}}}(t, s)$ according to the discrete feedback from Lemma 5.4 satisfy for all $s \in \mathbb{P}^{d-1}$

$$
\mid h \sum_{i=0}^{\infty} \beta^{i} g\left(\varphi_{\tilde{F}_{\mathbb{P}}}(i h, s), \tilde{F}_{\mathbb{P}}\left(\varphi_{\tilde{F}_{\mathbb{P}}}(i h, s)\right)-\tilde{v}_{\delta}^{h}(s) \mid \leq \frac{2 \eta}{\delta h}+C \frac{(h \varepsilon(h))^{\gamma}}{h} .\right.
$$

Proof: Abbreviate

$$
K(s):=h \sum_{i=0}^{\infty} g\left(\varphi_{\tilde{F}_{\mathbb{P}}}(i h, s), \tilde{F}_{\mathbb{P}}\left(\varphi_{\tilde{F}_{\mathbb{P}}}(i h, s)\right) .\right.
$$

From the construction of the control it follows that

$$
\tilde{v}_{\delta}^{h}(s)=h g\left(s, \tilde{F}_{\mathbb{P}}(s)\right)+\beta \tilde{v}_{\delta}^{h}\left(\tilde{\varphi}_{1}\left(s, \tilde{F}_{\mathbb{P}}(s)\right)\right)+2 \eta(s)
$$

where $|\eta(s)|<\eta$. On the other hand it holds that

$$
K(s)=h g\left(s, \tilde{F}_{\mathbb{P}}(s)\right)+\beta K\left(\varphi\left(h, s, \tilde{F}_{\mathbb{P}}(s)\right)\right) .
$$

Putting these two equations together yields

$$
\begin{aligned}
& \left|K(s)-\tilde{v}_{\delta}^{h}(s)\right| \\
= & \beta\left|K\left(\varphi\left(h, s, \tilde{F}_{\mathbb{P}}(s)\right)\right)-\tilde{v}_{\delta}^{h}\left(\tilde{\varphi}_{1}\left(s, \tilde{F}_{\mathbb{P}}(s)\right)\right)+2 \eta(s)\right| \\
\leq & \beta\left|K\left(\varphi\left(h, s, \tilde{F}_{\mathbb{P}}(s)\right)\right)-\tilde{v}_{\delta}^{h}\left(\varphi\left(h, s, \tilde{F}_{\mathbb{P}}(s)\right)\right)\right|+2 \eta+C(h \varepsilon(h))^{\gamma}
\end{aligned}
$$

and the assertion follows.

To obtain the main theorem we simply have to put together the estimates of this section. 
Theorem 5.6. Consider the projected system (2.4) and the optimal value function $v_{\delta}^{h}$ from (3.2). Let $h>0$ be a given time step. Then for every $\varepsilon>0$ there exists $\eta>0$ and $\varepsilon(h)>0$ such that the discrete feedback $\tilde{F}_{\mathbb{P}}$ from Lemma 5.4 satisfies

$$
\left|\int_{0}^{\infty} e^{-\delta t} g\left(\varphi_{\tilde{F}_{\mathbb{P}}}(t, s), F_{\mathbb{P}}\left(\varphi_{\tilde{F}_{\mathbb{P}}}\left(\left[\frac{t}{h}\right] h, s\right)\right)\right) d t-v_{\delta}^{h}(s)\right|<\varepsilon
$$

for all $s \in \mathbb{P}^{d-1}$ and the results from Section 4 also apply to $\tilde{F}_{\mathbb{P}}$.

Proof: Follows from Lemma 5.1, Lemma 5.2, Theorem 5.3 and Proposition 5.5 .

REMARK 5.7. Using the results from Section 4 it follows from this theorem that for any accessible semilinear system that is open loop exponentially asymptotically null controllable a stabilizing discrete feedback can be computed numerically. The main limitation for practical purposes lies in the numerical effort that is necessary to obtain sufficiently accurate solutions of the related optimal value functions - especially in higher dimensions.

REMARK 5.8. The accuracy $\varepsilon(h)$ from (5.1) needed for the numerical approximation of the trajectories strongly depends on the continuity properties of $\tilde{v}_{\delta}^{h}$. This is easily seen by looking at the second error term in estimate $(5.5)$.

In particular if $\tilde{v}_{\delta}^{h}$ is Lipschitz, (i.e. $\gamma=1$ ), a first order method for the calculation of $\tilde{\varphi}_{i}$ (i.e. $\varepsilon(h)=O(h)$ ) is sufficient to obtain convergence. Although Lipschitz continuity of $\tilde{v}_{\delta}^{h}$ could be observed in many numerical examples even for small $\delta>0$, up to now it is not clear if this property can be proved analytically.

\section{A Numerical Example}

In this section we will apply the numerical algorithm to a four-dimensional system (cp. [22]). This semilinear control system consists of two linear oscillators (in the $\left(x_{1}, x_{2}\right)$ plane and in the $\left(x_{3}, x_{4}\right)$ plane, respectively) that are coupled by the control term.

$$
\left(\begin{array}{c}
\dot{x}_{1} \\
\dot{x}_{2} \\
\dot{x}_{3} \\
\dot{x}_{4}
\end{array}\right)=\left(\begin{array}{cccc}
0 & 1 & 0 & 0 \\
-1-u & -0.2 & -u & 0 \\
0 & 0 & 0 & 1 \\
-u / \sqrt{2} & 0 & -2-u / \sqrt{2} & -0.2 \sqrt{2}
\end{array}\right)\left(\begin{array}{l}
x_{1} \\
x_{2} \\
x_{3} \\
x_{4}
\end{array}\right)
$$

Note that for the given choice of parameters the matrix $A(u)$ has only eigenvalues with positive real parts for all $u \in[-0.6,0.6]$. Hence for any constant control function $u(\cdot) \equiv u \in[-0.6,0.6]$ the system is unstable. Furthermore $[2$, Theorem 1 (i)] implies that there is no continuous feed back law stabilizing the system.

The following figures show some trajectories for this system by their projection into the $\left(x_{1}, x_{3}\right)$ plane. The arrows indicate the direction of the trajectories.

The Figures (1) - (3) show some trajectories for constant control values. Figure (4) and Figure (5) show trajectories of the system stabilized by a discrete feedback for different initial values. The Lyapunov exponent of 
these trajectories is -0.052 which is even smaller than the approximation of $\tilde{\kappa}$ by $\delta \tilde{v}_{\delta}^{a}$ which is -0.03 . This means that the numerical approximation still contains some significant error; nevertheless the accuracy is sufficient to calculate a stabilizing discrete feedback.

All trajectories have been computed using the extrapolation method for ordinary differential equations by Stoer and Bulirsch [23, Section 7.2.14]. The discretization of the trajectory $\tilde{\varphi}$ for the numerical calculation of the discrete feedback has been done by the Euler method, which was sufficiently accurate since the value function is Lipschitz, cp. Remark 5.8. The parameters of the discretization were $\delta=h=0.01$ and the approximation $\tilde{v}_{\delta}^{a}$ of $\tilde{v}_{\delta}^{h}$ has been computed as described in [14] on an equidistant grid with 8000 nodes. It turned out that for the stabilization it is sufficient to use the extremal control values $u=-0.6$ and $u=0.6$. 


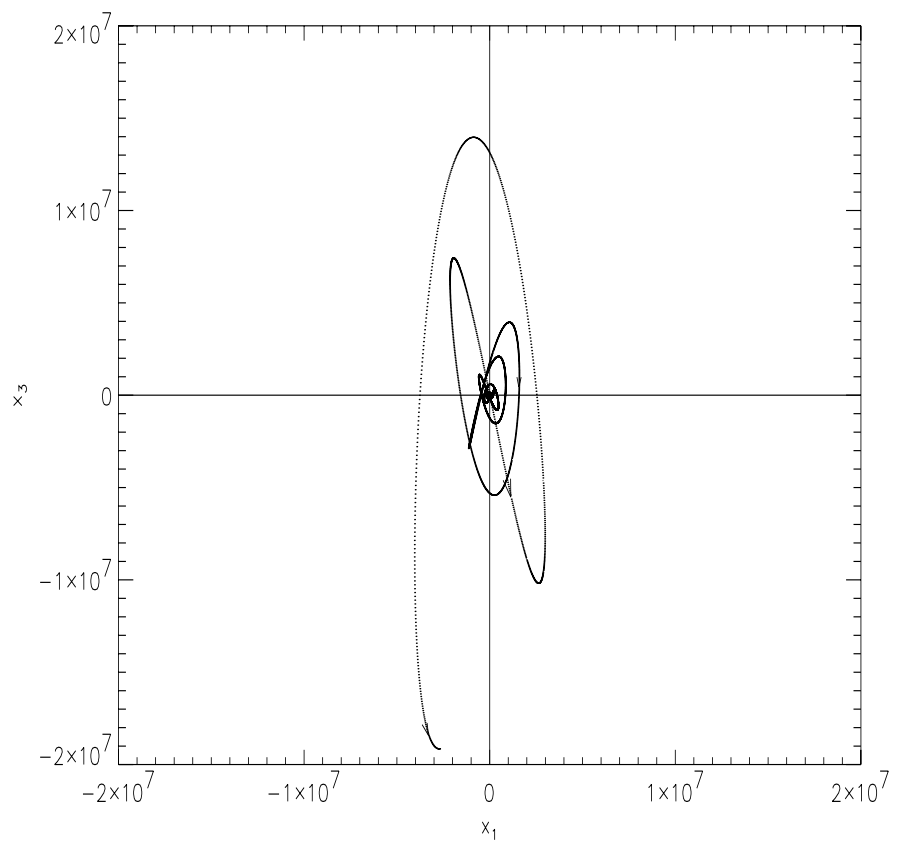

Figure 1. Trajectory for $u \equiv 0, x_{0}=\left(\begin{array}{llll}1 & 1 & 0.1 & 0.1\end{array}\right)^{T}$

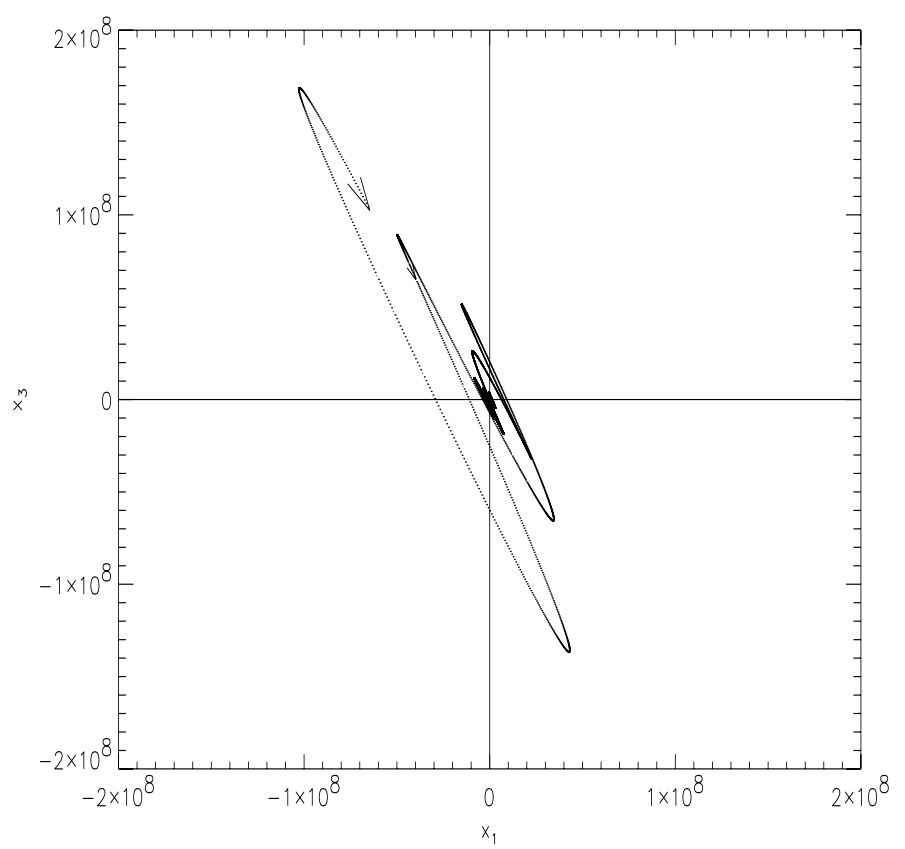

Figure 2. Trajectory for $u \equiv-0.6, x_{0}=\left(\begin{array}{llll}1 & 1 & 0.1 & 0.1\end{array}\right)^{T}$ 


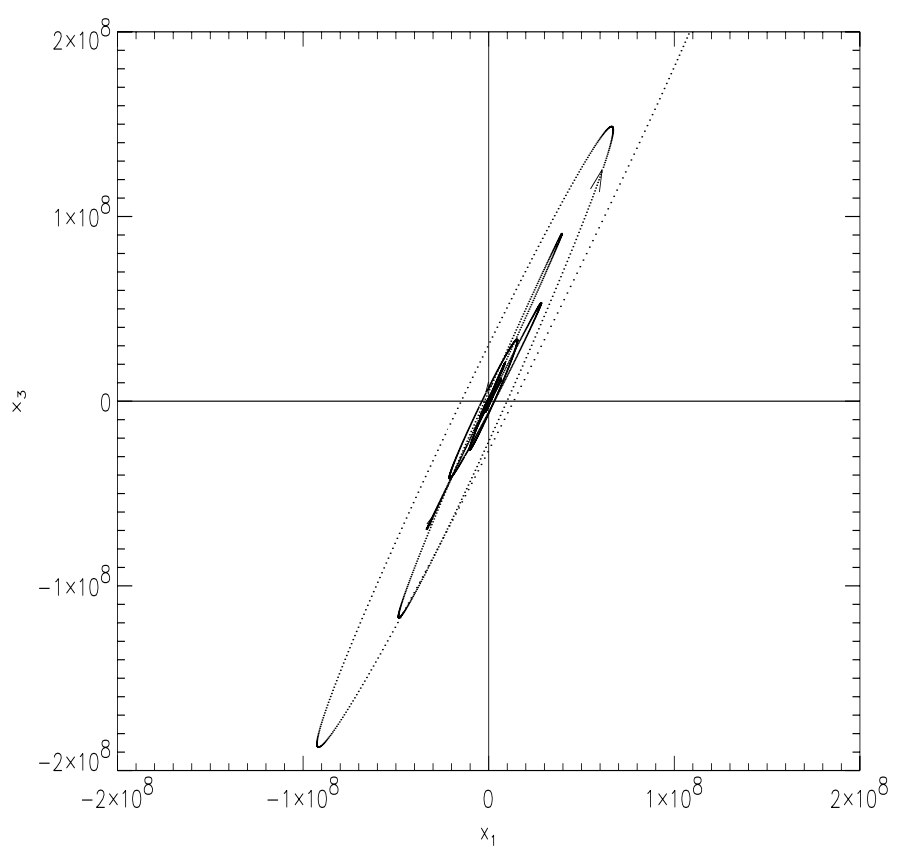

Figure 3. Trajectory for $u \equiv 0.6, x_{0}=\left(\begin{array}{llll}1 & 1 & 0.1 & 0.1\end{array}\right)^{T}$

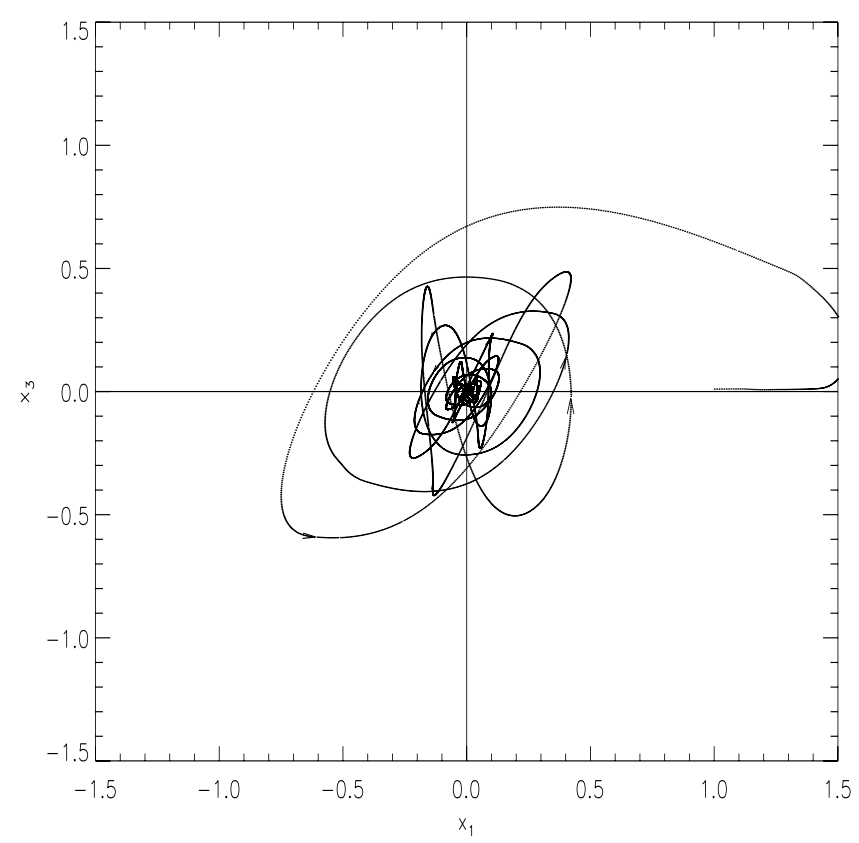

Figure 4. Trajectory with discrete feedback, $\mathrm{U}=[-0.6,0.6]$, $x_{0}=\left(\begin{array}{llll}1 & 1 & 0.1 & 0.1\end{array}\right)^{T}$ 


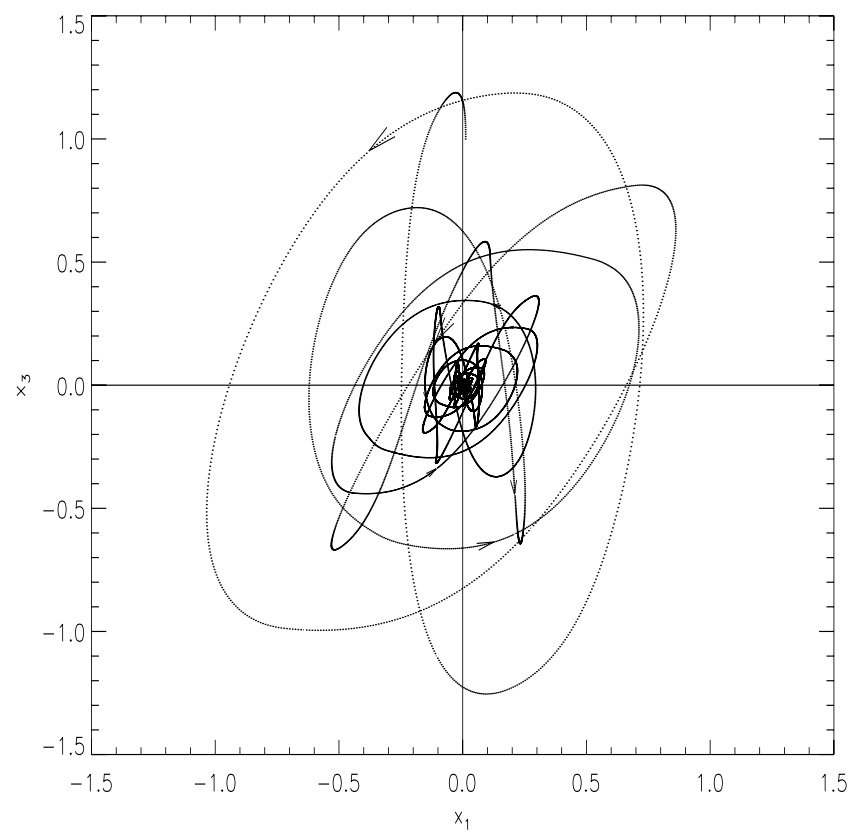

Figure 5. Trajectory with discrete feedback, $\mathrm{U}=[-0.6,0.6]$, $x_{0}=\left(\begin{array}{llll}0.1 & 0.1 & 1 & 1\end{array}\right)^{T}$

I would like to thank Fritz Colonius for his constant help and advice.

\section{REFERENCES}

[1] A. Bacciotti: Local Stabilizability of Nonlinear Control Systems, World Scientific, Singapore, 1992.

[2] R. W. Brockett: Asymptotic stability and feedback stabilization, in Differential Geometric Control Theory, R.W. Brockett, R.S. Millman, and H.J. Sussmann, eds., 181191, Birkhäuser, Boston, 1983.

[3] I. Capuzzo Dolcetta and M. Falcone: Discrete dynamic programming and viscosity solutions of the Bellman equation, Ann. Inst. Henri Poincaré, Anal. Non Linéaire, 6 (supplément), 1989, 161-184.

[4] I. Capuzzo Dolcetta and H. Ishii: Approximate solutions of the Bellman equation of deterministic control theory, Appl. Math. Optim., 11, 1984, 161-181.

[5] R. Chabour, G. Sallet, and J. Vivalda: Stabilization of nonlinear systems: A bilinear approach, Math. Control Signals Syst., 6, 1993, 224-246.

[6] F. Clarke, Y. Ledyaev, E. Sontag, and A. Subbotin: Asymptotic controllability and feedback stabilization, in Proc. Conf. on Information Sciences and Systems (CISS 96), Princeton, NJ, 1996. To appear, full version submitted.

[7] B. D. Coller, P. Holmes, and J. L. Lumley: Control of bursting in boundary layer models, Appl. Mech. Rev., 47, 1994, 139-143.

[8] F. Colonius and W. Kliemann: Linear control semigroups acting on projective space, J. Dyn. Differ. Equations, 5, 1993, 495-528.

[9] - Maximal and minimal Lyapunov exponents of bilinear control systems, $J$. Differ. Equations, 101, 1993, 232-275.

[10] - Asymptotic null controllability of bilinear systems, in Geometry in Nonlinear Control and Differential Inclusions, Banach Center Publications Vol. 32, Warsaw, 1995, 139-148. 
[11] M. Falcone: A numerical approach to the infinite horizon problem of deterministic control theory, Appl. Math. Optim., 15, 1987, 1-13. Corrigenda, ibid. 23, 1991, 213214.

[12] M. Falcone and R. Ferretti: Discrete time high-order schemes for viscosity solutions of Hamilton-Jacobi-Bellman equations, Numer. Math., 67, 1994, 315-344.

[13] L. Grüne: An adaptive grid scheme for the discrete Hamilton-Jacobi-Bellman equation, Numer. Math., 1996. To appear.

[14] - Numerical stabilization of bilinear control systems, SIAM J. Control Optim., 1996. To appear.

[15] H. Hermes: On stabilizing feedback attitude control, J. Optimization Theory Appl., 31, 1980, 373-384.

[16] - On the synthesis of stabilizing feedback control via Lie algebraic methods, SIAM J. Control Optim., 18, 1980, 352-361.

[17] A. Isidori: Nonlinear Control Systems: An Introduction, Springer Verlag, Berlin, 1989.

[18] N.N. Krasovskiŭ and A.I. Subbotin: Game-Theoretical Control Problems, Springer Verlag, New York, 1988.

[19] P.-L. Lions: Generalized solutions of Hamilton-Jacobi equations, Pitman, London, 1982.

[20] E.D. Sontag: Nonlinear regulation: The piecewise linear approach, IEEE Trans. Autom. Control, AC-26, 1981, 346-358.

[21] E.D. Sontag: Feedback stabilization using two-hidden-layer nets, IEEE Trans. Neural Networks, 3, 1992, 981-990.

[22] N. Sri Namachchivaya and H.J. Van Roessel: Maximal Lyapunov exponents and rotation numbers for two coupled oscillators driven by real noise, J. Stat. Phys., 71, 1993, 549-567.

[23] J. Stoer and R. Bulirsch: Introduction to Numerical Analysis, Springer Verlag, New York, 1980. 